\title{
Wearable Bio and Chemical Sensors
}

\author{
Shirley Coyle ${ }^{1}$, Vincenzo F. Curto ${ }^{1}$, Fernando Benito-Lopez ${ }^{1,2}$, \\ Larisa Florea ${ }^{1}$, and Dermot Diamond ${ }^{1}$
}

${ }^{1}$ INSIGHT: Centre for Data Analytics, National Centre for Sensor Research, Dublin City

University, Dublin, Ireland, ${ }^{2} \mathrm{CIC}$ microGUNE, Arrasate-Mondragón, Spain

\section{INTRODUCTION}

Chemical and biochemical sensors have experienced tremendous growth in the past decade due to advances in material chemistry combined with the emergence of digital communication technologies and wireless sensor networks (WSNs) [1]. The emergence of wearable chemical and biochemical sensors is a relatively new concept that poses unique challenges to the field of wearable sensing. This is because chemical sensors have a more complex mode of operation, compared to physical transducers, in that they must interact in some manner with specific molecular targets in the sample medium. To understand the challenges in developing wearable chemical and biochemical sensors the traits of these devices will be discussed in this introductory section. Following this the potential parameters of interest are presented and examples of wearable systems are discussed. A range of sampling techniques and methods of chemical sensing are presented along with integration issues and design challenges. Finally, some of the main application areas of this novel technology are discussed.

\subsection{Chemical and Biochemical Sensors}

Typically, a chemical or biochemical sensor consists of a recognition element (receptor) coupled with a transduction element (Figure 1). In the receptor part, the chemical information, e.g., the concentration of a given compound, $\mathrm{pH}$, etc., is converted into a form of energy that can be measured by a transducer. The function of the receptor is fulfilled in many cases by a thin layer that is able to interact with the analyte molecules, catalyze a reaction selectively, or participate in a chemical equilibrium together with the analyte [2]. 


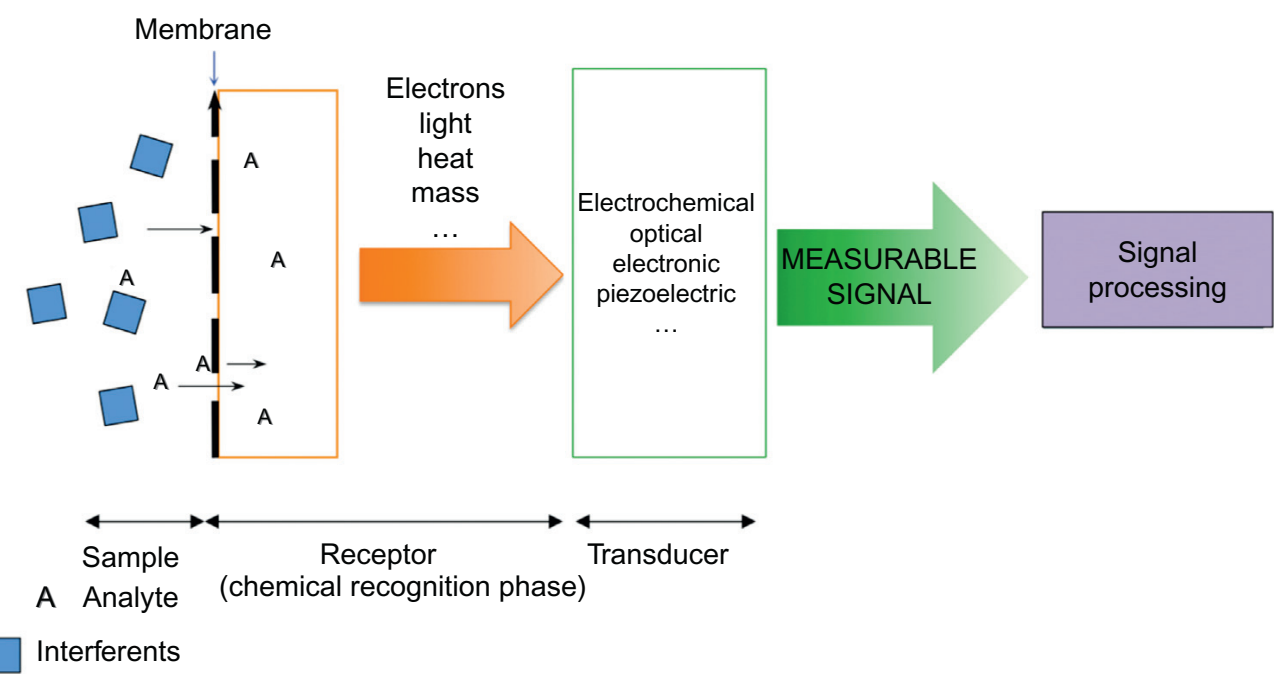

FIGURE 1 Schematic representation of the working principle of a chemical (biochemical) sensor. Some details of a real sensor are indicated. As an example, a filter or membrane may be used to separate the analyte to be detected from the interfering particles.

The transducer translates the chemical information about the sample into a useful analytical signal. The transduction element may be electrochemical, optical, electronic, or piezoelectric in nature. In recent years, novel chemical and biochemical sensors have been developed by scientific groups all over the world. This is evident by the growing number of international scientific articles in this area.

In spite of the clear advances in sensing technologies and high number of laboratory prototype sensors that appear every year, only a small fraction of these sensors reach commercialization. Apart from the complexity of the receptor design, the main reason for this is the incompatibility of the sensor platform with the real-world application. Sensors exposed to real-life scenarios are subjected to many environmental effects that can affect stability, reproducibility, and sensitivity. Effective sampling methods are crucial to avoid contamination and ensure controlled delivery to the active sensor surface. For example, a chemical sensor analyzing a body fluid such as sweat, blood, or tears must collect and deliver a sample to the sensor's active surface, whereupon a binding event happens and a signal is generated [3]. Other issues relate to system integration, sensor miniaturization, and low-power sensor interface circuitry design. One possible route toward the development of new and improved chemical sensing technologies is the emergence of nanoscience, related new nanostructured materials and multi-functional polymers. It is therefore not surprising that the last decade has witnessed rapid development in the field of chemical and biochemical sensors in parallel with the development of new materials [4].

Several chemical and biochemical wearable sensors have been developed in recent years, and they include $\mathrm{pH}$ sensors for sweat, sensors for several electrolytes (sodium, chloride, potassium) in sweat, the oximeter sensor, and others; however, most of them require further optimization and assessment in clinical trials before exploitation and routine use can occur. 


\subsection{Parameters of Interest}

The gold-standard fluid for diagnostics is blood, however, blood analysis requires invasive sampling, which is undesirable for long-term continuous use. In recent years, significant effort has been focused on sampling and analysis of alternative body fluids, such as interstitial fluid [5], tears [6], saliva [7], and sweat [8]. The transition from blood to other body fluids provides a less invasive means of sampling, which in turn may provide a route to facilitate longer-term continuous monitoring.

Sweat is probably the most accessible fluid to be collected by a garment [9]. Many relevant physiological analytes such as sodium, chloride, potassium, calcium, ammonia, glucose, and lactate are normally present in sweat. The sweat test of sodium and chloride concentrations is the gold-standard technique for the diagnosis of cystic fibrosis [10]. Analysis of sweat loss and sweat composition can also offer valuable information regarding hydration status and electrolyte balance, which can be especially important during sports activities.

Saliva is another large accessible bodily fluid present in the oral cavity, in which it is possible to get access to a large number of analytes and biomarkers. Saliva contains ions such as sodium, potassium, chloride, bicarbonate, nitrates, urea, uric acid, creatinine, and more than 400 types of proteins [11]. However, the use of saliva can be quite difficult due to the presence of mucus and residual food and blood, which can interfere with sensor operation.

Breath analysis can reveal a diverse signature of physiological processes that occur in the body. Conventional methods of breath analysis typically involve gas chromatography coupled with a form of mass spectroscopy [12]. These techniques require complex and expensive equipment not conducive to wearable formats. Chemical sensor arrays or electronic noses (e-noses) are an alternative cheaper, portable, and faster approach that have shown promising results in the fields of renal disease, lung cancer, and diabetes [13]. With these methods breath analysis still has challenges regarding standardization of methods of collection, treatment, and conditioning of samples.

Tears are another body fluid in which many proteins and electrolytes are normally present [14]. Glucose is one of the most important biomarkers present in tears, and may be useful in personal monitoring of diabetes. Recently, glucose in interstitial fluids also gained much attention for the realization of wearable sensors, and in particular through the use of microneedles, as discussed in more detail in section 2.12.

Wound exudate may be analyzed to monitor the healing process by monitoring different cell types, growth factors, and nutritional factors. Temperature and $\mathrm{pH}$ of the wound are also recognized to be critical for healing. For instance, Voirin et al. [15] realized an optical-integrated wound sensor using $\mathrm{pH}$ responsive hydrogels to provide information on the wound-healing process.

In addition to monitoring samples from the body, wearable chemical sensors may be used to sense substances in the environment around the wearer and thereby warn of any hazards that may be present. This has great implications for those working in challenging or extreme conditions such as first responders and military personnel. Detection of gases that are dangerous to health such as carbon monoxide or potential explosive agents can obviously help to ensure safety. 


\section{SYSTEM DESIGN}

This section focuses on the components of a wearable chemical or biochemical sensor system. A sample, either a body fluid or a gas, must be collected and delivered in the first instance. Then a suitable sensing mechanism must be integrated within the sensing platform also incorporating associated electronics and power supply. This overall system must be designed so that it can be placed on the body in a suitable location for long-term use in a reliable and comfortable manner.

\subsection{Sample Handling}

Micro-fluidics have significantly contributed to the development of personalized healthcare and point-of-care (POC) diagnostic devices. Micro-fluidic systems can deal with micro-volumes of the target sample and generate exactly the same information as standard analytic techniques [16]. This smaller sample volume of body fluid reduces the size and improves the reliability of the fluid-handling system. Another advantage is the possibility of integrating the active sensing area inside the micro-fluidic channel to minimize sample dead-volume, and consequently also the delay between sampling and analysis. For a wearable configuration, textile compatible methods are preferable. One approach is to use low or zero-power passive pumps based on capillary force driven micro-fluidics. This is discussed in the following section.

\subsubsection{Transport of Fluids in a Textile}

The wetting and wicking properties of natural and synthetic fibers can provide efficient means for controlling fluid flows without the need for external pumps. A recent example of a textile-based interfacial micro-fluidic platform was reported by Xing et al. [17], in which a 3D micro-fluidic network was fabricated by "stereo-stitching" of cotton yarn on a superhydrophobic textile. Taking advantage of the difference in wettability between the cotton yarn (contact angle $=0^{\circ}$ ) and the superhydrophobic textile (contact angle $=140^{\circ}$ ), a surface tension-driven micro-fluidic system was created in which the flow was inversely proportional to the flow resistance of the hydrophilic yarn. Under these conditions, the interfacial micro-fluidic can operate in two different regimes, the discrete transport mode and the continuous transport mode (Figure 2).

Other interesting examples of fabric-based micro-fluidics presented by Nilghaz et al. [18] were both two-dimensional and three-dimensional cloth-based devices achieved through the deposition of hydrophobic wax, which confined the flow within the wax border in a similar manner to paper-based micro-fluidic technology. The patterned fabric is capable of retaining its flexibility and mechanical strength, which in turn makes it suitable for future use in wearable technologies. Other approaches using hydrophobic and hydrophilic silk yarns have been proposed by Bhandari et al. [19]. They demonstrated the use of a handloom machine, but with the potential to be scaled up for mass production as it uses a well-established manufacturing technique within the textile industry.

A textile-based fluidic system was developed under the EU BIOTEX project to collect and analyze sweat on the body in real-time [20]. The system employed a passive pumping 
(a)

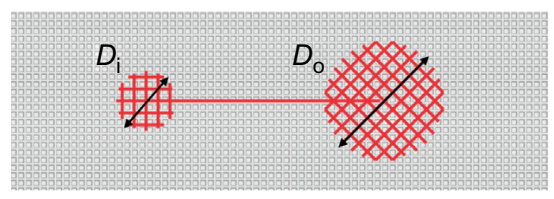

Cross-sectional view

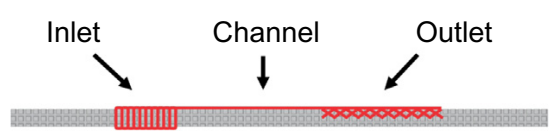

(b)

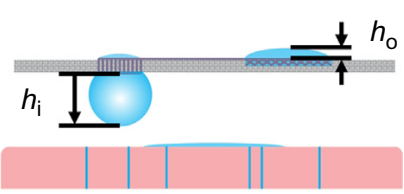

(c)

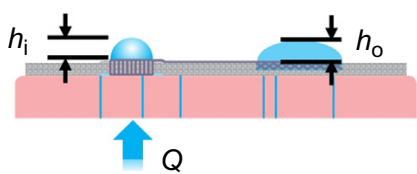

FIGURE 2 Textile-based interfacial micro-fluidics platform. (a) Top view and crosssectional view of the interfacial micro-fluidics in which the gray layer represents the superhydrophobic textile, while the hydrophilic cotton yarn is in red. Schematic representation of the (b) discrete transport mode and (c) continuous transport mode regimes (image courtesy of Siyuan Xing).
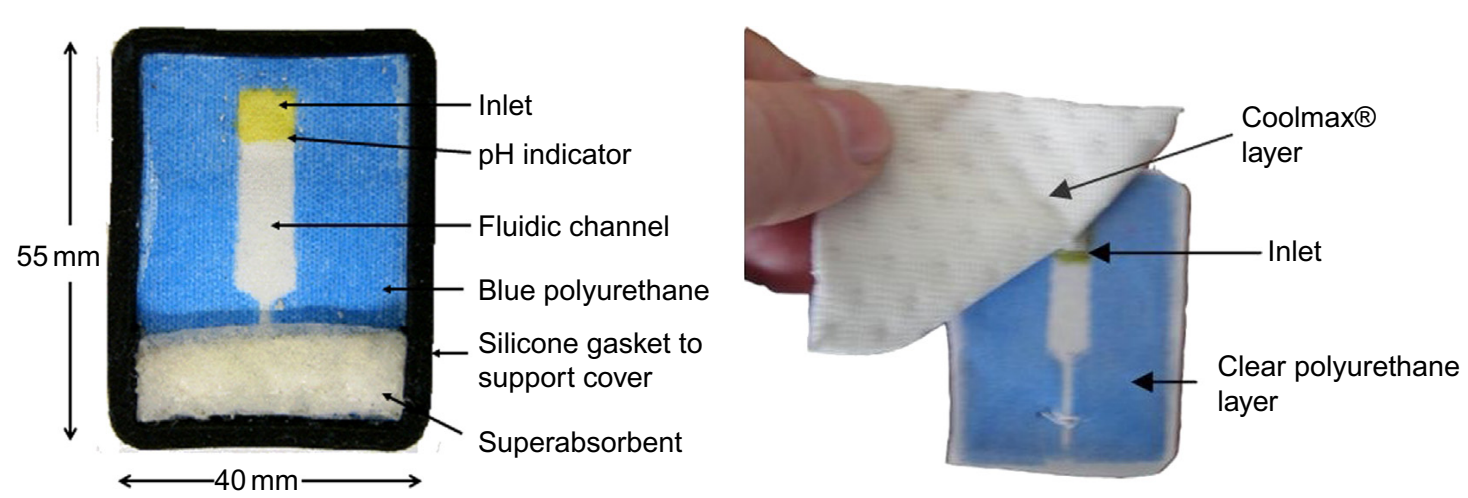

FIGURE 3 Textile-based platform to collect and analyze sweat developed during EU BIOTEX project.

mechanism based on capillary action using a combination of moisture-wicking fabric and a highly absorbent material. A fabric fluidic channel was formed by screenprinting a hydrophobic material (polyurethane) on either side of a polyester/Lycra ${ }^{\circledR}$ blend. An acquisition layer of fabric (Coolmax $\AA /$ / polyester/polyester) was stitched at this inlet to maximize sweat collection over a larger area (Figure 3). Sensing elements were placed in contact with the sample in the channel, including a dye for colorimetric analysis of $\mathrm{pH}$ and electrochemical sensors printed onto a Kapton film [21]. It was found that a priming time of 10 to 20 minutes was needed for the $\mathrm{pH}$ sensor, and around 35 minutes for the conductivity/sodium sensor due to the dead volume of the platform. A key goal in wearable chemical sensor platform design should therefore be to minimize dead volume and thereby reduce the delay between sampling and analysis.

Further to the development of the BIOTEX platform, Curto et al. [22] developed a miniaturized flexible plastic micro-fluidic system based on the same principle. The microfluidic device was capable of drawing fresh sweat from the skin surface to the detection area using a cotton thread and superabsorbent inside a microfluidic channel. The authors also demonstrated the use of this device coupled with a wireless platform for sweat $\mathrm{pH}$ 


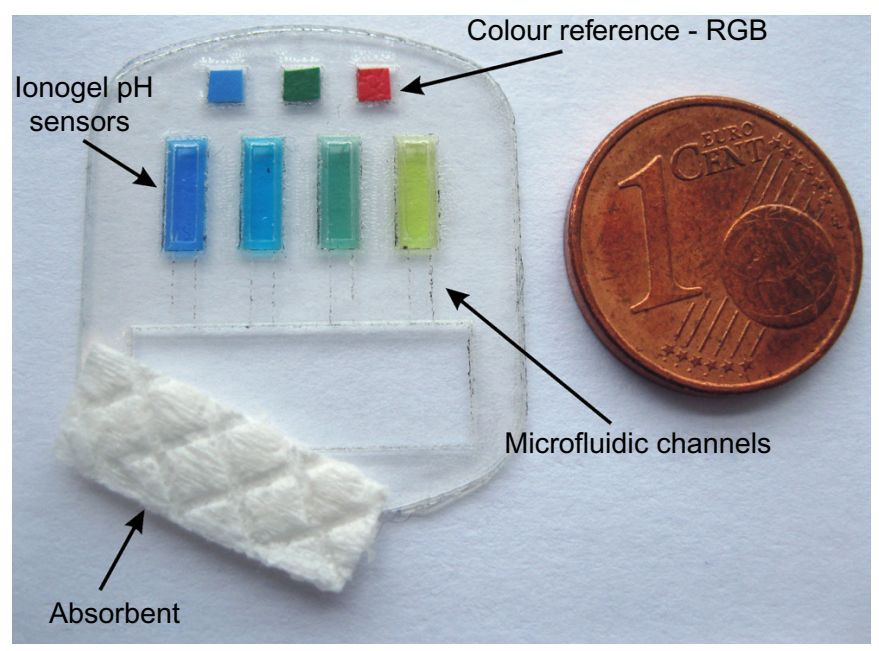

FIGURE 4 Wearable micro-fluidic barcode for $\mathrm{pH}$ analysis of sweat.

monitoring over a period of 50 minutes during cycling activity. Parallel to this, an alternative approach was developed using an electronic-free visual device [23]. The device presents four different sensing areas made of an ionogel (ionic liquids encapsulated in a cross-linked polymeric gel matrix) doped with four different $\mathrm{pH}$ dyes, forming a barcodetype sensor array. Three colored reference patches (Red, Green, and Blue - RGB) were incorporated at the top of the device to allow for automatic correction of ambient light (Figure 4). The color change and $\mathrm{pH}$ can be estimated by eye, or more accurately, by using a digital image or smartphone application.

These wearable sensors were mainly developed for single-use applications since integration of textile bio-chemical sensors into clothes that can be used multiple times and for long periods of time is still very challenging. These sensors are subject to the same problems of other common physical sensors like environmental noise and motion artifacts, but are also subject to additional constraints that come from the nature of the sensing mechanism itself (chemical or biochemical reaction, changes in the sensor surface, contact of sensor surface with analyte, etc.). In addition, for everyday use of the textile sensors, external factors like sensor-surface contamination, which decreases the accuracy of the sensor, need to be considered. Moreover, since the sensors are ideally integrated into clothes, the possibility of washing the sensors needs to be taken into account; therefore the sensing area needs to be somehow protected or the sensor has to be washing-compatible.

\subsubsection{Microneedle Technology}

Microneedle arrays offer a minimally invasive means of biosensing through highly integrated biocompatible devices. There is the possibility of making them wearable through small devices and patches directly in contact with the skin. The trend is to make these arrays easy to fabricate on an industrial scale and at low cost.

The principal applications of these microneedle arrays involve fluid sampling [24] and extraction [25] since they provide the opportunity to overcome the skin barrier and thereby reach dermal biofluids, which are more reflective of systemic levels of key 
analytes. This novel technology can be used as a therapeutic tool for transdermal drug delivery, including insulin, and more recently as a diagnostic tool for the analysis of the biofluid contents. In this regard, glucose is being intensely investigated using microneedles in interstitial fluid. For instance, Sakaguchi et al. [26] developed a sweat-monitoring patch for measuring glucose using a minimally invasive interstitial fluid extraction technology based on microneedles. The advantage of this technique is that sweat contamination during interstitial fluid glucose extraction is avoided. Good correlations were found between interstitial fluid and reference plasma glucose levels.

Other analytes measured using microneedle sampling were hydrogen peroxide and ascorbic acid in which sampling was integrated with sensing via chemically modified carbon fiber bundles [27], and lactate, using carbon paste microneedle arrays. In the latter case, the need for integrated microchannels was avoided and extraction of the interstitial fluid was avoided, as the microneedles themselves were provided with inherent sensing capabilities [28]. Highly linear lactate detection was achieved over the entire physiological range, along with high selectivity, sensitivity, and stability of the carbon paste microneedle array. Recently, Miller et al. [29] reported the use of similar microneedle configurations for the simultaneous detection of multiple analytes in physiologically relevant tissue environments. The microneedles selectively detected changes in $\mathrm{pH}$, lactate, and glucose, showing their potential use for applications in sports science. Biopotential measurements were demonstrated by OMahony et al. [30] using microneedles as dry electrodes for detecting electrocardiography (ECG) and electromyography (EMG). The microneedle-based dry electrodes compared to conventional wet electrodes are shown in Figure 5.

The fabrication of functional wearable devices that incorporate microneedle technology is very challenging. Nowadays, micro-fluidics are becoming the most reliable approach to control fluid transport in these sensors systems, where in many cases, only small sample volumes may be available (e.g., interstitial fluid flow is lower than $10 \mu \mathrm{L} \mathrm{h}^{-1}$ ). In this regard, Strambani et al. [31] developed a silicon microchip for transdermal injection/sampling applications. The microneedles were connected with a number of independent reservoirs integrated in the back side of a silicon die. Flow rate through the needle array as a
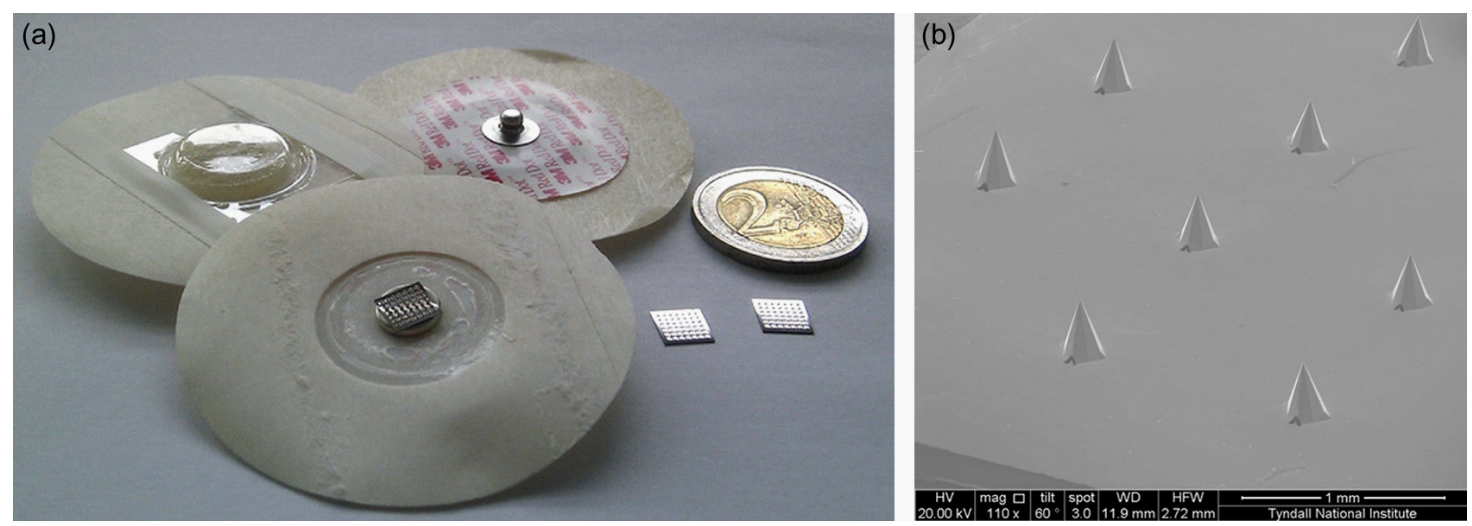

FIGURE 5 (a) Traditional wet electrode (top left), assembled prototype electrodes (bottom left), and microneedle arrays (right). (b) Microneedle-based dry electrode, illustrating an array of microneedles [30]. 
function of the pressure drop applied to the chip for injection/drawing purposes was investigated. Using an array with 38,000 active needles, the flow rate can be finely controlled from a few $\mathrm{mL} \mathrm{min}{ }^{-1}$ up to tens of $\mathrm{mL} \mathrm{min}^{-1}$. Other considerations that need to be taken into account when using microneedle arrays for in vivo sensing are the clogging of the microneedles and the potential of structural deformation upon their insertion into the skin, which may change the dynamics of the sampling and thereby introduce an unpredictable delay from time of sampling to detection.

\subsubsection{Sampling Gases}

Gas sensors may be employed to monitor the air surrounding the wearer (e.g., to detect dangerous levels of toxic gases). Delivery of the gas sample to the sensing surface can be controlled by choosing a fabric of suitable permeability to encase the sensor. Such a textile layer would also serve to protect the sensing surface against pollution and mechanical damage. In addition to the choice of fabric, the location of wearable gas sensors on the body needs careful consideration (e.g., depending on the density of the gas being detected compared to air).

Another possible wearable gas sensor is a breath sensor. However, collection of breath samples in a wearable system is challenging to do in an unobtrusive yet accurate manner. An approach taken by the portable Lapka ${ }^{\mathrm{TM}}$ breath alcohol monitor [32] is to use a small cylindrical device to collect the sample with the hand closed around it as a mouthpiece. The device is a ceramic cylinder $(57 \mathrm{~mm} \times 23 \mathrm{~mm}$ ) with embedded sensors, which are wirelessly connected to a mobile device with an associated app. Perhaps in the future a headset-based design with an app, similar to the one created by BreathResearch [33] for measuring breath acoustics, capable of measuring chemical and biochemical parameters in breath, would be feasible.

\subsection{Types of Sensors}

There are various sensing techniques that can be employed for producing wearable chemical and biochemical sensors, and this influences the platform design and thus the materials and electronics required for integration into a textile or wearable device. Colorimetric and electrochemical sensing have both been demonstrated for wearable chemical and biochemical sensing, and examples of each will be discussed in this section.

\subsubsection{Wearable Colorimetric Sensing Platforms}

Colorimetric sensors involve a color change at the active sensor surface, which can then be measured using optical techniques. One way of combining sensing with a textile fluidic system is to use colorimetric methods. Nilghaz et al. [34] have summarized recent progress in the development of micro-fluidic devices based on multifilament threads and textiles for clinical diagnostic and environmental sensing.

A colorimetric approach to $\mathrm{pH}$ measurement in sweat using $\mathrm{pH}$ sensitive dyes and surface mount LEDs and photodiodes has been developed by the Adaptive Sensors Group at Dublin City University, as discussed in section 2.1.1. Li et al. [35] first demonstrated the application of thread-based micro-fluidic devices for the development of low-cost colorimetric diagnostic sensors. The capillary wicking of a fabric remarkably improves fluid 
transport characteristics, and therefore such devices can be readily integrated into clothing like T-shirts through the knitting of chemical-sensitive yarns within the fabric. In fact, Reches et al. [36] further explored the concept of thread micro-fluidics, showing the possibility of performing multiplexed colorimetric analysis through the use of a single thread sewn through a wearable adhesive plaster. This has the potential for sensing a wide range of analytes, such as proteins, ketones, and nitrates.

A wearable colorimetric biosensor within a contact lens has been developed to detect glucose levels in tears. Badugu et al. [37] demonstrated the use of disposable contact lenses embedded with boronic acid-based fluorophores for the colorimetric detection of glucose. The contact lens changes color according to the amount of glucose in tears, and is monitored by the wearer by simply looking into a mirror and comparing the color to a precalibrated color strip (Figure 6).

\subsubsection{Electrochemical}

Electrochemical sensors involve using electrodes to measure the electrochemical changes that occur when chemicals interact with a sensing surface. Recently, a great deal of effort has been applied to the development of wearable electrochemical sensors through the employment of novel materials, such as conductive polymers and carbon nanomaterials. For instance, carbon nanomaterials present excellent electrical and chemical properties, and they have been extensively explored for the realization of biosensors.

Guinovart et al. [38] developed potentiometric sensors for the detection of $\mathrm{pH}$, potassium, and ammonium levels in sweat using cotton yarns coated with single-walled carbon nanotubes (SWCNTs). The sensors were realized by dip-coating the yarns inside a SWCNTs aqueous solution used as ink, followed by further integration of the ion-selective membrane. These devices exhibited reproducible responses over a period of two months, being used once a week. The integration of the sensor into a band-aid for real-time detection of potassium on a manikin was demonstrated, although, as the authors well highlighted, the incorporation of the sensor into a truly wearable platform would require further investigation. Also using a potentiometric sensor, Schazmann et al. [8] developed a wearable platform to perform realtime measurements of sodium concentration in sweat. A liquid contact ion selective electrode (ISE) was integrated within a sodium sensor belt (SSB), which was then applied to the comparison of sodium concentration in sweat emitted by healthy and cystic fibrosis-positive people during exercise. However, for wearable potentiometric ISE sensors, solid contact ISE (scISE) are preferable to classic liquid contact ISE. In fact, scISEs can be easily fabricated on
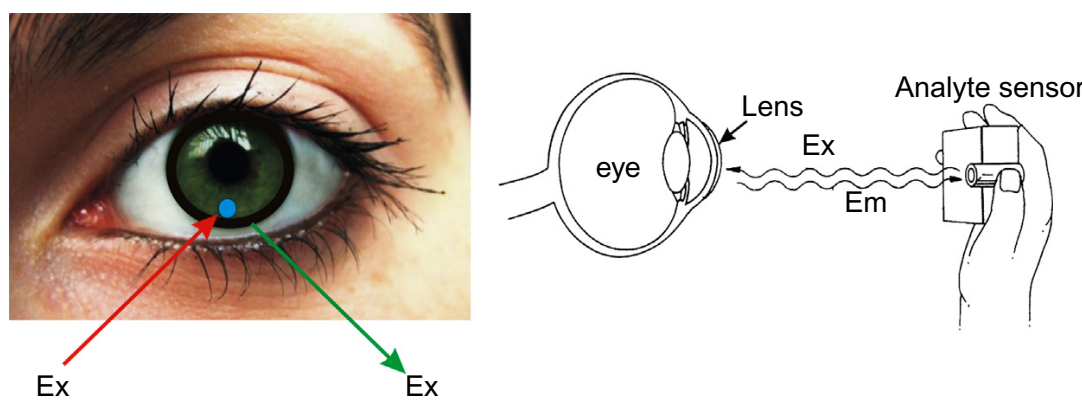

FIGURE 6 Continuous tear glucose monitoring using bored doped contact lenses (left). The hand held device works by flashing a light into the eye (Ex) and measuring the emission (Em) intensity. Schematic representation of the tear glucose-sensing device (right). Adapted from [37]. 
all-plastic flexible substrates, which in turn makes them more compatible with wearable technology, as they can be fabricated in a more conformal fashion. Following this approach, Bandodkar et al. [39] demonstrated real-time epidermal $\mathrm{pH}$ monitoring via a tattoo-based scISE. The sensor was fabricated using screen printing technology in the form of a "smiley face," in which the eyes are, respectively, the reference electrode and the scISE, the latter incorporating a $\mathrm{pH}$-sensitive layer made of polyaniline-conductive polymer. Interestingly, the same concept can be readily expanded for the development of similar epidermal ISE tattoosensors for the detection of other ionic species present in sweat, such as sodium, potassium, chloride, and ammonium, through the use of appropriate selective membranes for the targeted ionic analyte. Using a similar approach, Jia et al. [40] fabricated a flexible printed temporary-transfer tattoo electrochemical biosensor that could be used for the real-time detection of lactate in human perspiration. The biosensor was tested when applied to the deltoid of an athlete for real-time lactate monitoring during cycling, and showed good correlation with parallel in vitro evaluation of sweat samples collected during the exercise. Khodagholy et al. [41] also demonstrated a conformal organic electrochemical transistor (OECT) incorporating an ionogel for the electrochemical determination of lactate in sweat. The use of OECTs for lactate sensing represents an interesting and novel strategy for future integration of this biosensor into a wearable platform. There are several advantages to the use of OECTs compared to more conventional sensor platforms, such as easy integration into electronic circuits and operation at low applied bias compared to traditional field-effect transistors (FET). In addition to this, OECTs can be entirely made of Poly(3,4-ethylenedioxythiophene) Polystyrene sulfonate (PEDOT:PSS) conductive polymer, which makes them very low cost and compatible with industrial stamping processes, such as roll-to-roll technology.

Yang et al. [42] demonstrated that electrochemical sensors could be printed onto clothing for the realization of wearable biosensors. Carbon strips were printed directly on the elastic waistband of underwear, offering a tight and direct contact with the skin. The sensors were capable of maintaining electrochemical behavior after several cycles of folding or stretching of the supporting textile, indicating the potential application of this sensor configuration for wearable electrochemical chemo-/biosensors on clothes.

Claramunt et al. [43] have developed a flexible sensor array using bare and metal-decorated carbon nanofibers as an active layer for on-body gas sensing. Inkjet printing was used to deposit four interdigited silver electrodes onto a Kapton film, and a heater was printed onto the reverse side of the film. Inkjet printing has the advantage of being non-contact and is suitable for many substrates. Metal-decorated carbon nanofibers were spray coated onto the electrodes and the sensor response was characterized for ammonia and nitrogen dioxide. Carbon nanotubes were also demonstrated for sensing gases and biological molecules [44,45].

\section{CHALLENGES IN CHEMICAL BIOCHEMICAL SENSING}

\subsection{Sensor Stability}

Ideally, a wearable sensor should be autonomous and reliable over time. However, long-term stability of chemical sensors and biosensors in autonomous operation remains a 
major issue. These sensors typically need an active (responsive) surface to operate, and changes to this surface due to fouling or leaching of reagents can lead to baseline drift and variations in sensitivity. There may also be a cross-response to interferents that may be present in the sample. Therefore, there is a need for chemical and biochemical sensors to be frequently calibrated, which requires liquid handling for sampling, reagents, and waste. Consequently, systems must include pumps, valves, and liquid storage, which are clearly impractical in terms of current technologies for autonomous wearable devices. This is one of the main reasons that there are few practical systems at present. One approach is to develop low-cost disposable sensors. In this way, part of the system may be reusable, e.g., control electronics for measurement, detection, and wireless capability, but the sensor surface may be inserted/removed easily. For example, the removable/disposable element may be a small micro-fluidic chip. This approach also provides an attractive cost model in terms of commercialization of devices.

\subsection{Interface with the Body}

A recent survey on wearable biosensor systems for health showed that wearability was a major issue because sensor, battery, and on-body hardware size tend to be too bulky. Sensor systems rapidly become redundant if patients or clinicians do not want to work with them. A review of patients' and clinicians' preferences for non-invasive, body-worn sensor systems found that a body-worn sensor system should be compact, embedded, simple to operate, and should not affect daily behavior nor seek to directly replace a healthcare professional [46]. Therefore, wearable chemical sensors should be seen as an additional tool to assist in healthcare delivery and to provide better continuity of care.

Ideally, the sensor should be flexible, comfortable to wear, and lightweight. Miniaturization of sensors can help to reduce power requirements, and also makes the device less obtrusive for the wearer. Through device miniaturization, we also reduce the size of the sample needed for the sensor to operate.

In the case of sweat analysis, samples from a single site on the body may not give an accurate representation of the total body sweat loss as there are regional variations in sweat composition and volume. Therefore multiple sampling sites may be needed, and a study by Patterson et al. [47] has identified four sampling regions (the chest, scapula, forearm, and thigh) to estimate mean whole-body concentrations of electrolytes in sweat.

Technology is becoming more and more portable. Sensors, including imaging cameras, GPS, and accelerometers, are being increasingly integrated into mobile phones. This is stimulating the emergence of new apps for monitoring personal health. For example, pulse oximetry apps are now available based on wearable Bluetooth-enabled optical sensors on mobile phones, and research into colorimetric sensing is increasing. The smartphone may become part of a wearable sensor system serving as the detection element, and also for data management and communications. An example of the smartphone being used for colorimetric analysis is presented by Oncescu et al., in which sweat and saliva $\mathrm{pH}$ measurements are taken using $\mathrm{pH}$-sensitive paper inserted into a smartphone accessory [48]. An example of the data management capability of smartphones is the iBGStar [49] by Sanofi Diabetes, which allows users to track and manage their blood glucose levels by linking to 
an iPhone. Through mobile communications, this information can be shared by email, websites, or text messages to facilitate a movement toward remote management of personal health.

Increasingly, the smart watch is being considered as an interface to the mobile phone, and is facilitating integration of other wearable sensors. The great advantage of the watch form is that it is something that people are familiar with and used to wearing. An example of a wrist-worn device to monitor chemical parameters is a device for transdermal alcohol sensing by Giner, Inc. The device known as WrisTAS ${ }^{\mathrm{TM}}$ (Wrist Transdermal Alcohol Sensor) is a wrist-worn prototype to objectively track patterns of human alcohol consumption [50].

Newly developed electronic "tattoo" technology discussed previously in section 2.2.2 is a non-invasive means of producing conformal and mechanically stable sensors that can be applied directly to the skin. These devices may be easily fabricated using well-established printing technologies, and have great potential for providing access to multi-parameter sensing platforms. For example, MC10 [51] is developing body-conformable electronics that are stretchable and bendable. Figure 7 shows the biostamp technology that is capable of measuring physiological signals such as body temperature and heart rate.

\subsection{Textile Integration}

Section 2.1.1 presented various approaches to handling body fluids using textile yarns and fabrics. However, for textile-based chemical sensors to become a practical reality, their incorporation must be compatible with traditional manufacturing techniques such as weaving and lamination. For analysis of body fluids, the fluid-handling and sensing elements must both be compatible in order to create a real system that can be used on the body and yield valid results for real-time measurements at an acceptable cost.

Two groups in Switzerland, Ecole Polytechnique Fédérale de Lausanne (EPFL) and Eidgenoessiche Technische Hochschule Zurich (ETHZ), have demonstrated the

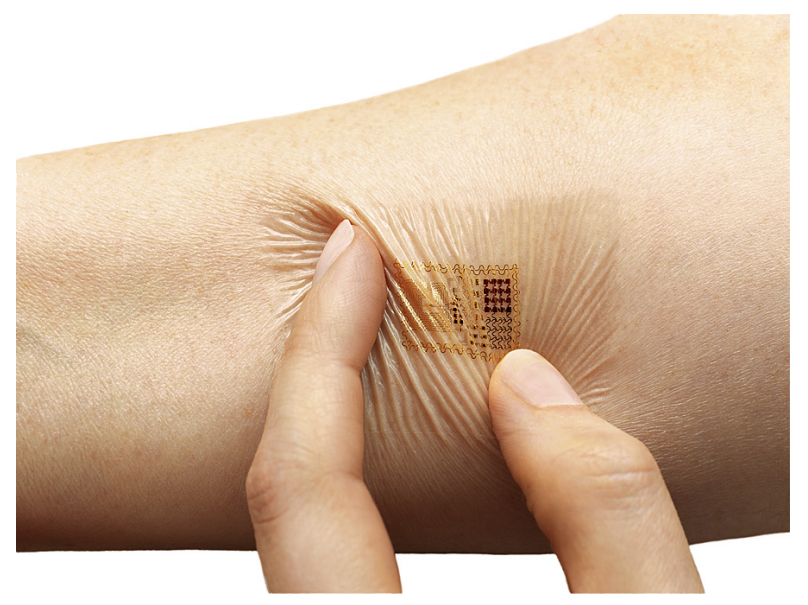

FIGURE 7 Biostamp technology developed by MC10 Inc. (photo courtesy of mc10 Inc.) 


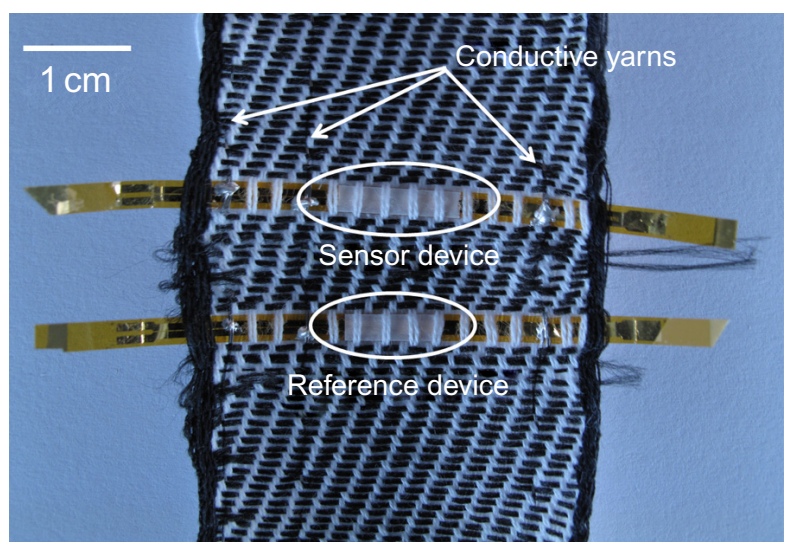

FIGURE 8 Sensing components on thin strips of Kapton film woven into fabric (photo courtesy of Ecole Polytechnique Fédérale de Lausanne, EPFL).

integration of gas sensors into a woven textile. Sensing components on thin strips $(25 \mathrm{~mm})$ of Kapton film have been integrated into the weaving process as shown in Figure 8 . The sensing regions were well encapsulated and found to retain functionality after weaving and repeated stress/strain testing [52]. The sensing element is an interdigited thin-film capacitor coated with a humidity-sensitive polymer (cellulose acetate butyrate). Changes in ambient humidity caused changes in the capacitance of the sensing layer. In principle, this humidity-sensitive polymer layer could be replaced by a gassensitive polymer to target other analytes. Troester's group in ETHZ has demonstrated the use of carbon black/polymer sensors in a similar woven Kapton film configuration to detect vapor such as acetone [53].

\subsection{Power Requirements}

Limited battery life, size, and weight are the most significant inconveniences for most wearable electronic devices. Low-power device performance should be the first priority through smart integration and miniaturization of the electronic components. To power these devices using lightweight, comfortable components, flexible batteries, energyharvesting methods, or remote charging may be considered.

Practical miniature devices that can harvest sufficient kinetic energy from the human body to power a wireless bio-sensor are still in their infancy. Energy-harvesting feasibility depends mainly on four factors: the typical power consumption of the device; the use pattern; the device size (and thus the acceptable harvester size); and the motion to which the device is subjected. All these factors need to be considered in order to generate an autonomous, self-powering, wearable device for monitoring body parameters.

Chemical sensors based on RFID tags have been demonstrated as passive devices requiring no power supply to be connected to the sensor. An RFID tag typically consists of a microchip attached to a radio antenna. An RFID reader transmits signals to the RFID tag and reads the signal sent back from the tag. By creating a sensing film on the antenna of an RFID tag, the analyte-induced changes can affect the impedance of the antenna and 
hence the resonant frequencies. GE Global Research has demonstrated this principle and performed RFID sensing in water to investigate the levels of ions and organic solvents, and in gases to detect toluene and ammonia [54]. More recent work has involved the development of self-correction methods against fluctuations in ambient temperature [55]. McAlpine's group at Princeton University used an external radio transmitter to power their tooth "tattoo" sensor used to monitor respiration and bacteria in saliva [56]. However, this sensing technology is still in its early stages as adhesion to the tooth, along with sampling issues, still need to be investigated further.

\section{APPLICATION AREAS}

\subsection{Personal Health}

Wearable sensors are applicable for all stages of treatment in healthcare, including prevention, immediate care, rehabilitation, and long-term support. If it is possible to find a link between non-invasive or minimally invasive chemical/biochemical detection methods and well-established but invasive blood testing, then a new range of tools may be created for the healthcare industry.

Diabetes is a chronic condition that involves close monitoring of glucose levels, typically using finger-prick samples. Menarini Diagnostics have developed a continuous monitor for blood glucose measurement called the GlucoDay ${ }^{\circledR}$ S [57]. The device uses a microdialysis probe inserted into the abdomen for continuous sampling of interstitial fluid. The GlucoDay ${ }^{\circledR} \mathrm{S}$ is specifically for clinical use and is worn by the subject for a 48 hour period. Such continuous monitoring can show general trends that may be missed by single-point measurements. The data generated by the GlucoDay ${ }^{\circledR} S$ is the equivalent of approximately 1,000 finger-prick glucose values. The main goal for the device is to chart an individual's daily glucose activity, especially during sleeping hours. With this information the healthcare team and the patient can modify the insulin regimen in order to achieve better control patterns.

Wearable chemical and biochemical sensors have the potential to improve the quality of life in the healthcare arena through real-time assessment. In terms of market statistics, the biggest market is for sensors that can "sense" and "monitor" key diagnostic and therapeutic markers. In the U.S. alone, the Sensors Market in Healthcare Applications is expected to Reach $\$ 13.11$ billion by 2017 [58].

\subsection{Sports Performance}

Technology has a huge role to play in improving sports performance, from textiles to simulate a shark's skin gliding through the water for swimwear to moisture-wicking fabrics keeping athletes comfortable and cool. Physiological monitoring has helped our understanding of the body and its response to exercise, and through physiological measurement individual training regimes can be planned and optimized. During prolonged periods of training, diverse physiological conditions can be easily reached. The most commonly monitored parameter in sports science is blood lactate, and while there are portable systems 
available for this measurement, it is still an invasive approach, giving point measurements from blood samples. Continuous monitoring via a non-invasive approach would be far more favorable. However, it will be necessary to investigate the correlations between the levels of critical parameters in blood compared to other body fluids if this is to provide useful information. In a review of several studies, the conclusion was that the relationship between blood lactate and lactate in other body fluids is unclear [59].

Real-time sweat analysis during exercise could give valuable information on dehydration and changes in the amount of important biomolecules and ions. Dehydration increases perceived effort, so if exercise feels hard, then people may be less inclined to partake in it. In the case of elite athletes, dehydration can greatly impact performance. Sweat electrolyte concentration varies greatly between individuals, being affected by genetics, sweat rate, type of training, degree of hydration, and the state of heat acclimatization. The opportunity of gathering important information on the physical condition of athletes during sport activities could potentially provide huge benefits, arising from personalized training regimes and optimized rehydration/nutrition strategies.

\subsection{Safety and Security}

There are many scenarios in which personal safety could be supported through the use of wearable sensors for continuous monitoring of external hazards. Some high-risk professions include first responders, construction workers, police, security, and military personnel. Wearable sensors for these applications must of course be lightweight, ergonomic, robust, and accurate. Working in a demanding environment, they need to be easy to operate and ideally part of a wider sensor network. In addition to personal alerts, communication between team members is needed for management and localization of overall team safety.

An EU project, ProeTEX, is focused on the safety of firefighters through the integration of toxic gas sensors among other sensing capabilities into protective clothing [60]. Carbon monoxide $(\mathrm{CO})$ and carbon dioxide $\left(\mathrm{CO}_{2}\right)$ sensors were integrated into the jacket and boots of a firefighter's uniform. Figure 9 shows a $\mathrm{CO}_{2}$ sensor integrated into the boot of a firefighter's uniform and a $\mathrm{CO}$ sensor integrated close to the jacket collar. The choice of placement was based on the densities of each gas. The $\mathrm{CO}$ and $\mathrm{CO}_{2}$ gas sensors already have an outer protective membrane built in to filter out small particles (dust, soot, etc.). On the garment, the gas-sensing devices are further protected by full encapsulation into a purposely designed pocket with a heat resistance, waterproof, airpermeable membrane, hence protecting them from potential damage from heat, water, and airborne particles.

Researchers at Arizona State University have developed a wearable sensor to monitor air quality. The device communicates with a smartphone for user feedback and is suitable for indoor and outdoor environmental personal exposure studies. In a recent study [60] the device was used to test air quality in three scenarios: indoors after room remodeling and painting, outdoors near a busy highway and, finally, outdoors in areas affected by an oil spill. Such a sensor has the potential to greatly improve our knowledge of personal exposures and to help protect human health as well as the environment. 
(a)

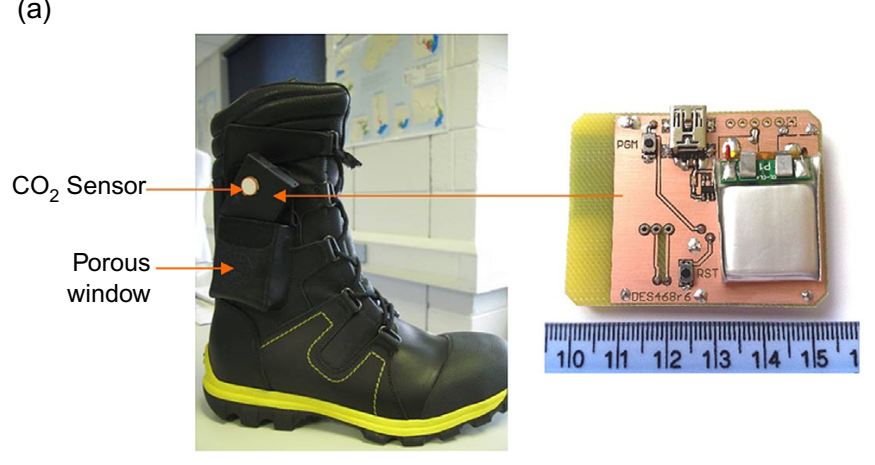

(b)

FIGURE 9 Wearable chemical sensing in ProeTEX project (a) Carbon dioxide sensor integrated into a firefighter's boot and (b) carbon monoxide sensor integrated into a firefighter's jacket.

\section{CONCLUSIONS}

Smart fabrics and interactive textiles are a relatively new area of research with many potential applications in the field of biomedical engineering. The ability of smart textiles to interact with the body provides a novel means to sense the wearer's physiology and respond to the needs of the wearer. The advantage of this technology relies on the integration of sensors in clothes that are worn on a daily basis, providing the capacity to continuously monitor the wearer and his/her environment. To date, most of the research in the field of wearable sensing has focused on physical sensors that respond to changes in their immediate environmental proximity (e.g., electric fields, heat, and movement). Relatively little has been done on the development of similar wearable sensors for real-time monitoring of chemical and biological parameters of interest. The main reasons for this are due to the fact that these sensors require an active surface at which a chemical reaction occurs, which generates the observable signal. However, this surface is subjected to samples, reagents, waste products, and contaminants from the environment that can affect its functionality. Sample handling requires additional hardware such as fluidic systems with valves and pumps, and for this requirement micro-fluidics could play an important role. Furthermore, wearability is probably the most important requirement that these sensors must fulfill without losing functionality and, above all, compromising the wearer's comfort and/or safety.

It is clear that wearable chemical sensors have the potential to provide a means to continuously monitor the physiology of the wearer over extended periods of time, in an 
innocuous manner that does not interfere with the daily routines of wearers. Through this technology, the wearer can be kept informed of his/her well-being in a dynamic manner, and individuals can become actively involved in the management of their personal health, making pHealth (pervasive health) a feasible goal. There is also great potential in the field of sports science, particularly in the real-time analysis of sweat during exercise. The other main application area of wearable chemical and biochemical sensors is in personal safety in warning of hazardous chemicals in the environment, which is of particular importance to first responders in disaster situations, and those involved in security and safety. This chapter has detailed a summary of the state of the art while also highlighting the current issues and challenges for the future of this exciting field of research.

\section{Acknowledgment}

This review work was supported by the Science Foundation Ireland under Insight award SFI/12/RC/2289 and the Gobierno Vasco, Dpto. Industria, Innovación, Comercio y Turismo under ETORTEK 2012 with Grant No. IE12-328, ETORTEK 2013 with Grant No. IE13-360. FBL thanks the Ramón y Cajal programme (Ministerio de Economía y Competitividad), Spain.

\section{References}

[1] R. Byrne, F. Benito-Lopez, D. Diamond, Materials science and the sensor revolution, Mater. Today vol. 13 (2010) 9-16.

[2] A. Lobnik, M. Turel, Š. Korent Urek, Optical Chemical Sensors:Design and Applications, Advances in Chemical Sensors, InTech, 2012.

[3] S. Coyle, Y. Wu, K.-T. Lau, D. De Rossi, G. Wallace, D. Diamond, Smart Nanotextiles: A Review of Materials and Applications, MRS Bulletin vol. 32 (2007) 434-442.

[4] M. Gerard, A. Chaubey, B.D. Malhotra, Application of conducting polymers to biosensors, Biosens. Bioelectron. vol. 17 (2002) 345-359.

[5] K. Rebrin, N.F. Sheppard, G. Steil, Use of subcutaneous interstitial fluid glucose to estimate blood glucose: revisiting delay and sensor offset, J. Diabetes Sci. Technol. vol. 4 (2010) 1087-1098.

[6] M. Chu, T. Shirai, D. Takahashi, T. Arakawa, H. Kudo, K. Sano, et al., Biomedical soft contact-lens sensor for in situ ocular biomonitoring of tear contents, Biomed. Microdevices vol. 13 (2011) 603-611.

[7] E. Papacosta, G.P. Nassis, Saliva as a tool for monitoring steroid, peptide and immune markers in sport and exercise science, J. Sci. Med. Sport vol. 14 (2011) 424-434.

[8] B. Schazmann, D. Morris, C. Slater, S. Beirne, C. Fay, R. Reuveny, et al., A wearable electrochemical sensor for the real-time measurement of sweat sodium concentration, Anal. Methods vol. 2 (2010) 342-348.

[9] S. Coyle, F. Benito-Lopez, T. Radu, K.T. Lau, D. Diamond, Fibers and fabrics for chemical and biological sensing, J. Text. App vol. 14 (2010).

[10] J. Massie, K. Gaskin, P. Van Asperen, B. Wilcken, Sweat testing following newborn screening for cystic fibrosis, Pediatr. Pulmonol. vol. 29 (2000) 452-456.

[11] D.P. Lima, D.G. Diniz, S.A.S. Moimaz, D.r.H. Sumida, A.C.u. Okamoto, Saliva: reflection of the body, Int. J. Infect. Dis. vol. 14 (2010) e184-e188.

[12] K.-H. Kim, S.A. Jahan, E. Kabir, A review of breath analysis for diagnosis of human health, Trends Anal. Chem. vol. 33 (2012) 1-8.

[13] D. Hill, R. Binions, Breath Analysis for Medical Diagnosis, Int. J. Smart Sens. Intell. Syst. vol. 5 (2012) $401-440$.

[14] Y. Ohashi, M. Dogru, K. Tsubota, Laboratory findings in tear fluid analysis, Clin. Chim. Acta. vol. 369 (2006) $17-28$.

[15] G. Voirin, J. Luprano, S.p. Pasche, S. Angeloni, R.a. Ischer, M. Liley, Wearable biosensors for monitoring wound healing, Adv. Sci. Technol. vol. 57 (2009) 80-87. 
[16] G. Whitesides, Solving problems, Lab Chip vol. 10 (2010) 2317-2318.

[17] S. Xing, J. Jiang, T. Pan, Interfacial microfluidic transport on micropatterned superhydrophobic textile, Lab Chip vol. 13 (2013) 1937-1947.

[18] A. Nilghaz, D.H.B. Wicaksono, D. Gustiono, F.A. Abdul Majid, E. Supriyanto, M.R. Abdul Kadir, Flexible microfluidic cloth-based analytical devices using a low-cost wax patterning technique, Lab Chip vol. 12 (2012) 209-218.

[19] P. Bhandari, T. Narahari, D. Dendukuri, Fab-Chips': a versatile, fabric-based platform for low-cost, rapid and multiplexed diagnostics, Lab Chip vol. 11 (2011) 2493-2499.

[20] D. Morris, S. Coyle, Y. Wu, K.T. Lau, G. Wallace, D. Diamond, Bio-sensing textile based patch with integrated optical detection system for sweat monitoring, Sens. Actuators B vol. 139 (2009) 231-236.

[21] S. Coyle, K. Lau, N. Moyna, D. Diamond, F. Di Francesco, D. Constanzo, et al., BIOTEX-Biosensing textiles for personalised healthcare management, IEEE Trans. Inf. Technol. BioMed. vol. 14 (2010) 364-370.

[22] V.F. Curto, S. Coyle, R. Byrne, N. Angelov, D. Diamond, F. Benito-Lopez, Concept and development of an autonomous wearable micro-fluidic platform for real time $\mathrm{pH}$ sweat analysis, Sens. Actuators B Chem. vol. 175 (2012) 263-270.

[23] V.F. Curto, C. Fay, S. Coyle, R. Byrne, C. O’Toole, C. Barry, et al., Real-time sweat pH monitoring based on a wearable chemical barcode micro-fluidic platform incorporating ionic liquids, Sens. Actuators B Chem. vol. 171-172 (2012) 1327-1334.

[24] S. Chandrasekhar, L.K. Iyer, J.P. Panchal, E.M. Topp, J.B. Cannon, V.V. Ranade, Microarrays and microneedle arrays for delivery of peptides, proteins, vaccines and other applications, Expert Opin. Drug Deliv. vol. 10 (2013) 1155-1170.

[25] H. Suzuki, T. Tokuda, K. Kobayashi, A disposable "intelligent mosquito" with a reversible sampling mechanism using the volume-phase transition of a gel, Sens. Actuators B vol. 83 (2002) 53-59.

[26] K. Sakaguchi, Y. Hirota, N. Hashimoto, W. Ogawa, T. Hamaguchi, T. Matsuo, et al., Evaluation of a minimally invasive system for measuring glucose area under the curve during oral glucose tolerance tests: usefulness of sweat monitoring for precise measurement, J. Diabetes Sci. Technol. vol. 7 (2013) 678-688.

[27] P.R. Miller, S.D. Gittard, T.L. Edwards, D.M. Lopez, X. Xiao, D.R. Wheeler, et al., Integrated carbon fiber electrodes within hollow polymer microneedles for transdermal electrochemical sensing, Biomicrofluidics vol. 5 (2011).

[28] J.R. Windmiller, N. Zhou, M.-C. Chuang, G. Valdes-Ramirez, P. Santhosh, P.R. Miller, et al., Microneedle array-based carbon paste amperometric sensors and biosensors, Analyst vol. 136 (2011) 1846-1851.

[29] P.R. Miller, S.A. Skoog, T.L. Edwards, D.M. Lopez, D.R. Wheeler, D.C. Arango, et al., Multiplexed microneedle-based biosensor array for characterization of metabolic acidosis, Talanta vol. 88 (2012) 739-742.

[30] C. O’Mahony, F. Pini, A. Blake, C. Webster, J. O’Brien, K.G. McCarthy, Microneedle-based electrodes with integrated through-silicon via for biopotential recording, Sens. Actuators A Phys. vol. 186 (2012) 130-136.

[31] L.M. Strambini, A. Longo, A. Diligenti, G. Barillaro, A minimally invasive microchip for transdermal injection/sampling applications, Lab Chip vol. 12 (2012) 3370-3379.

[32] < https://mylapka.com/bam >, (Last Accessed: 23.06.14).

[33] < http://www.breathresearch.com/ > , (Last Accessed: 23.06.14).

[34] A. Nilghaz, D.R. Ballerini, W. Shen, Exploration of microfluidic devices based on multi-filament threads and textiles: A review, Biomicrofluidics vol. 7 (2013) 051501.

[35] X. Li, J. Tian, W. Shen, Thread as a Versatile Material for Low-Cost Microfluidic Diagnostics, ACS Appl. Mater. Interfaces vol. 2 (2009) 1-6, 2010/01/27.

[36] M. Reches, K.A. Mirica, R. Dasgupta, M.D. Dickey, M.J. Butte, G.M. Whitesides, Thread as a Matrix for Biomedical Assays, ACS Appl. Mater. Interfaces vol. 2 (2010) 1722-1728, 2010/06/23.

[37] R. Badugu, J.R. Lakowicz, C.D. Geddes, A Glucose Sensing Contact Lens: A Non-Invasive Technique for Continuous Physiological Glucose Monitoring, J. Fluores vol. 13 (2003) 371-374.

[38] T. Guinovart, M. Parrilla, G.A. Crespo, F.X. Rius, F.J. Andrade, Potentiometric sensors using cotton yarns, carbon nanotubes and polymeric membranes, Analyst vol. 138 (2013) 5208-5215.

[39] A.J. Bandodkar, V.W.S. Hung, W. Jia, G. Valdes-Ramirez, J.R. Windmiller, A.G. Martinez, et al., Tattoo-based potentiometric ion-selective sensors for epidermal pH monitoring, Analyst vol. 138 (2013) 123-128.

[40] W. Jia, A.J. Bandodkar, G. Valdos-Ramirez, J.R. Windmiller, Z. Yang, J. Ramirez, et al., Electrochemical Tattoo Biosensors for Real-Time Noninvasive Lactate Monitoring in Human Perspiration, Anal. Chem. vol. 85 (2013) 6553-6560, 2013/07/16.

\section{WEARABLE SENSORS}


[41] D. Khodagholy, V.F. Curto, K.J. Fraser, M. Gurfinkel, R. Byrne, D. Diamond, et al., Organic electrochemical transistor incorporating an ionogel as a solid state electrolyte for lactate sensing, J. Mater. Chem. vol. 22 (2012) 4440-4443.

[42] Y.-L. Yang, M.-C. Chuang, S.-L. Lou, J. Wang, Thick-film textile-based amperometric sensors and biosensors, Analyst vol. 135 (2010) 1230-1234.

[43] S. Claramunt, O. Monereo, M. Boix, R. Leghrib, J.D. Prades, A. Cornet, et al., Flexible gas sensor array with an embedded heater based on metal decorated carbon nanofibres, Sens. Actuators B Chem. vol. 187 (2013) 401-406.

[44] I.V. Anoshkin, A.G. Nasibulin, P.R. Mudimela, M. He, V. Ermolov, E.I. Kauppinen, Single-walled carbon nanotube networks for ethanol vapor sensing applications, Nano Res. vol. 6 (2013) 77-86.

[45] J. Li, H. Ng, H. Chen, Carbon nanotubes and nanowires for biological sensing, first ed., Protein Nanotechnology, vol. 300, Humana Press, 2005, pp. 191-223.

[46] J.H.M. Bergmann, A.H. McGregor, Body-Worn Sensor Design: What Do Patients and Clinicians Want? Ann. Biomed. Eng. vol. 39 (2011) 2299-2312.

[47] M. Patterson, S. Galloway, M.A. Nimmo, Variations in regional sweat composition in normal human males, Exp. Physiol. vol. 85 (2000) 869-876.

[48] V. Oncescu, D. O'Dell, D. Erickson, Smartphone based health accessory for colorimetric detection of biomarkers in sweat and saliva, Lab Chip vol. 13 (2013) 3232-3238.

[49] < http://www.bgstar.com/web/ibgstar > , (Last Accessed: 23.06.14).

[50] < http://www.ginerinc.com/>, (Last Accessed: 23.06.14).

[51] < http://www.mc10inc.com>, (Last Accessed: 23.06.14).

[52] C. Ataman, T. Kinkeldei, G. Mattana, A. Vasquez Quintero, F. Molina-Lopez, J. Courbat, et al., A robust platform for textile integrated gas sensors, Sens. Actuators B Chem. vol. 177 (2013) 1053-1061.

[53] T. Kinkeldei, C. Zysset, N. Muenzenrieder, G. Troester, An electronic nose on flexible substrates integrated into a smart textile, Sens. Actuators B Chem. vol. 174 (2012) 81-86.

[54] R. A. Potyrailo, C. Surman, W. G. Morris, and S. Go," Selective detection of chemical species in liquids and gases using radio-frequency identification (RFID) sensors," in Solid-State Sensors, Actuators and Microsystems Conference, 2009. TRANSDUCERS 2009. International, 2009, pp. 1650-1653.

[55] R.A. Potyrailo, C. Surman, A passive radio-frequency identification (RFID) gas sensor with self-correction against fluctuations of ambient temperature, Sens. Actuators B Chem. vol. 185 (2013) 587-593.

[56] M.S. Mannoor, H. Tao, J.D. Clayton, A. Sengupta, D.L. Kaplan, R.R. Naik, et al., Graphene-Based Wireless Bacteria Detection on Tooth Enamel, Nat. Commun. vol. 3 (2012).

[57] < http://www.menarinidiag.co.uk/Products/continuous_glucose_monitoring > , (Last Accessed 23.06.14).

[58] Markets and Markets. (2013), World Sensors Market in Healthcare Applications.

[59] P.J. Derbyshire, H. Barr, F. Davis, S.P.J. Higson, Lactate in human sweat: a critical review of research to the present day, J. Physiol. Sci. vol. 62 (2012) 429-440.

[60] C. Chen, K.D. Campbell, I. Negi, R.A. Iglesias, P. Owens, N. Tao, et al., A new sensor for the assessment of personal exposure to volatile organic compounds, Atmos. Environ. vol. 54 (2012). 